\title{
Conceptions of function translation: obstacles, intuitions, and rerouting
}

\author{
Rina Zazkis $^{\mathrm{a}, *}$, Peter Liljedahl ${ }^{\mathrm{a}}$, Karen Gadowsky ${ }^{\mathrm{b}}$ \\ ${ }^{a}$ Faculty of Education, Simon Fraser University, 8888 University Drive, Burnaby V5A 1S6, BC, Canada \\ ${ }^{\mathrm{b}}$ Delta Secondary School, BC, Canada
}

\begin{abstract}
A horizontal translation of a function is the focus of this study. We examine the explanations provided by secondary school students and secondary school teachers to a translation of a function, focusing on the example of the parabola $y=(x-3)^{2}$ and its relationship to $y=x^{2}$. The participants' explanations focused on attending to patterns, locating the zero of the function, and the point-wise calculation of function values. The results confirm that the horizontal shift of the parabola is, at least initially, inconsistent with expectations and counterintuitive to most participants. We articulate possible sources of this perceived inconsistency and describe a pedagogical approach aimed at resolving it.
\end{abstract}

(C) 2003 Elsevier Inc. All rights reserved.

Keywords: Function; Transformation; Translation; Obstacle; Transformation of function; Horizontal translation

\section{Prologue (motivation and questions)}

Imagine the graph of (a) $y=x^{2}$. Now imagine the graph of (b) $y=(x-3)^{2}$. Check your imagination with the graphing device. If you are not surprised, it is probably because you have already explored the relationship between the two parabolas in the past. Most people conjecture that graph (b) will appear three units to the left of graph (a). The surprising result is that (b) is actually three units to the right of (a).

As students, we memorized this result as one of the counterintuitive facts of mathematics. As teachers, we attempted to explain this phenomenon to our students, often creating a conflict between their intuition and our explanations.

\footnotetext{
* Corresponding author. Tel.: +1-604-291-3662; fax: +1-604-291-3203.

E-mail address: zazkis@sfu.ca (R. Zazkis).
} 
As researchers, we became interested in how teachers and students deal with the phenomenon and what explanations they provide. Moreover, we wished to understand possible sources of students' "wrong" intuition and sought pedagogical solutions.

\section{Background}

Teaching, learning, or understanding of functions has been an important focus in mathematics education research in the past decades. However, within a variety of research reports focusing on functions, little attention has been given to the transformations of functions. A common treatment of transformations of functions in pre-calculus courses involves a consideration of a graph of a function $f(x)$ on a Cartesian plane. Functions $f(a x), f(-x), f(x)+k$, and $f(x+k)$ correspond to a dilation, reflection, vertical translation and horizontal translation of $f(x)$, respectively. Discussion of transformations of functions traditionally begins with a consideration of a parabola and then proceeds to graphs of other quadratic relations and other functions.

Eisenberg and Dreyfus (1994) conducted an extensive exploration on students' understanding of function transformations, focusing on visualization of transformations. They acknowledged the difficulty in visualizing a horizontal translation in comparison to a vertical one, suggesting that "there is much more involved in visually processing the transformation of $f(x)$ to $f(x+k)$ than in visually processing the transformation of $f$ to $f(x)+k$ " (p. 58).

Baker, Hemenway, and Trigueros (2000) have investigated the understanding of transformations of various functions from a perspective of Action-Process-Object-Schema (APOS) theory (for a description of APOS, see for example, Asiala et al., 1996 or Dubinsky \& MacDonald, 2001). They confirmed the observation that vertical transformations appear easier for the students than horizontal transformations. They explained this based on their theoretical perspective, claiming that "vertical transformations are actions performed directly on the basic functions, while horizontal transformations consist of actions that are performed on the independent variable of the function and further action is needed on the object resulting from the first action to get the result of the transformation" (Baker et al., 2000, p. 47). Furthermore, students' difficulty with function transformation was attributed, at least in part, to their incomplete understanding of the concept of function. Baker et al. agree with Eisenberg and Dreyfus in their observation that an object conception of function may be a prerequisite to the effective understanding of transformations of functions.

Borba and Confrey (1996) have presented a detailed case study of a 16-year-old student, Ron, working on transformations of functions in a computer based multi-representational environment. Their study intended to investigate vertical and horizontal translations, reflections around vertical and horizontal lines, and vertical and horizontal stretches of functions. Ron's attempts to interpret the horizontal translation of a parabola are presented as "problematic." This is followed by his investigation to coordinate visual actions with changes in other representations. Though it is not explicitly stated in this research report, we infer from the focus of their discussion that researchers perceived other transformations as less "problematic."

In this study, we focus on the difficulty — acknowledged in the aforementioned prior research presented by a horizontal translation of a function. Our goal is to investigate how students and teachers cope with this difficulty and to explore how it is possible to overcome or at least to reduce it. We examine the understanding of horizontal translation of functions in general, focusing on parabolas in particular. We explore how participants explain the perceived inconsistency presented by a horizontal translation 
and suggest a pedagogical approach to transformations of functions that addresses the mystery of graphs "moving in unexpected directions."

\section{Methodology}

Participants in this study were preservice secondary teachers $(n=15)$, practicing secondary teachers ( $n=16)$, and Grade 11/12 students $(n=10)$. The teachers were volunteers attending courses at the University in which this study was conducted. The students were volunteers referred by the participating teachers, all classified as having "above-average" ability.

In a clinical interview setting, all the participants were asked to predict, check and explain the relationship between the graph of $y=x^{2}$ and the graph of $y=(x-3)^{2}$. The interview protocol started by inviting the participants to sketch both graphs on the same coordinate system and to explain how they generated their sketch. The participants were then invited to compare their sketch to that of a graphing calculator. After the sketch was either confirmed or disconfirmed, the participants were asked to comment on the location of the graph $y=(x-3)^{2}$ relative to the graph of $y=x^{2}$, relating, where necessary, to the discrepancy between the sketch and the display of the graphing calculator, or to the discrepancy between the intuitive expectation and the "known" result. In addition, if the issue did not come up naturally, the participants were asked to discuss the graph of $y=x^{2}-3$ and compare it to $y=(x-3)^{2}$.

Their responses were audio recorded, transcribed, and analyzed. The analysis attended to (1) common trends in explanations, (2) attitudes towards perceived inconsistencies, and (3) differences between the groups of participants, that is, students versus teachers, and preservice teachers versus practicing teachers.

\section{Results}

The fact that the shape of $y=(x-3)^{2}$ is a parabola that is congruent to the canonical parabola $y=x^{2}$ was taken for granted by teachers and students alike. The fact that the graph of $y=(x-3)^{2}$ is a horizontal translation of $y=x^{2}$ was also mentioned as requiring no further explanation. Therefore, we turn our focus now to the direction of this horizontal translation.

\subsection{Students' responses}

The participating students had learned about transformations of quadratics (conic sections) before this study was conducted. Nevertheless, in the very beginning of the interview half of the students ( 5 out of 10) predicted the spatial location of $y=(x-3)^{2}$ incorrectly. There was no sincere attempt on students' part to explain the phenomenon, though all admitted that the observed location of the parabola was counterintuitive.

We chose to present in detail the following excerpt from the interview with Mitch, a Grade 11 student, because it captures most of the themes that appeared in students' responses. Mitch learned in class about transformations of functions and their corresponding graphs about two months prior to the interview. The interview excerpt presented below takes place after Mitch incorrectly sketched the graph of $y=(x-3)^{2}$. 
Interviewer: $\quad$ Would you please check this.

Mitch:

No problem. (pause) Yes problem. I should have remembered this. It moves in the wrong direction.

Interviewer: What do you mean, wrong direction?

Mitch:

It's minus three, so you sort of expect it to move left. But it moves the other way. It moves right.

Interviewer: $\quad$ Oh, and why is that?

Mitch:

That's the way it is. It's always like that. I really knew that. Just remember to do the opposite. But not always. Just when ... only if your number is in the brackets.

Interviewer: Please explain.

Mitch:

OK. When you do something to $x$ in the brackets, like $x$ minus three or $x$ plus two your graph moves the opposite way. For $x$ plus two it moves left, two times. For $x$ minus three, the one you wanted, it moves right, three times. And once you remember that, it works for all the graphs.

Interviewer: $\quad$ You mentioned something about brackets.

Mitch:

Yes, it moves the opposite way only when there are brackets. Without brackets, say for $y$ equals $x$ squared plus three, written this way, [ $\left.y=x^{2}+3\right]$, it moves the way you want it to move. This one will move up, and with negative three it will move down. This one is doing exactly what it should.

Interviewer: $\quad$ So the other one is doing something it shouldn't.

Mitch:

Not really, just not what one would guess, but the other way around.

Interviewer: Does it bother you?

Mitch:

Not really. You just see on the calculator what it's doing and you know it will do it all the time.

Interviewer: $\quad$ I wonder why the graph [of $y=(x-3)^{2}$ ] moves the way it moves. It's not what I expected, it's not what you expected, at least initially. Can you help me understand this?

Mitch: (pause). Maybe try small steps. Try $y$ equals $x$ minus one squared $\left[y=(x-1)^{2}\right]$. You see, it goes right one step. And try $y$ equals $x$ minus two squared $\left[y=(x-2)^{2}\right]$. It will go right two steps, you see them together here [demonstrates with graphing calculator]. So after seeing this you do not expect $x$ minus three $[(x-3)]$ to do something different, do you?

Interviewer: Is this how your teacher explained it to you?

Mitch:

I'm not sure he explained this at all. But graph it once and you know how it works.

In examining Mitch's response, we note the initial confusion, which is rapidly corrected based on the feedback from the graphing calculator. The claims "I should have remembered," "I knew that" demonstrate that the phenomenon is not new to Mitch: he has encountered this behavior of graphs earlier and expects to base his knowledge on this recollection. The claim "It moves in the wrong direction" and further clarification on what is meant by "wrong direction" presents a conflict between the result and the expectations; however, these expectations have been adjusted based on experience. Further, Mitch generalizes his experience of observing translations of the parabola ("once you remember that, it works for all the graphs") and also limits the scope of this observation ("only if your number is in the brackets"). Mitch explains that while the vertical translation matches his expectations, as "it moves the way you want 
it to move," the case of the horizontal translation in his approach is "just remember to do the opposite." However, Mitch is not troubled with the acknowledged counterintuitive behavior of $y=(x-3)^{2}$. His main concern appears to be with remembering this behavior, rather than with understanding it. Responding to the interviewer's quest to understand the location of the function, Mitch suggests the consideration of a pattern. Observing graphs of $y=(x-1)^{2}$ and $y=(x-2)^{2}$ implies the location of the graph of $y=(x-3)^{2}$. This explanation is consistent with his strong belief in internal consistency of this pattern — "graph it once and you know how it works."

As mentioned earlier, half of the students initially sketched the graph of $y=(x-3)^{2}$ incorrectly, that is, to the left rather than to the right of the canonic parabola $y=x^{2}$. The reaction of "I should have known" was common among these students. Participants who provided the correct sketch claimed that they recalled doing this in their mathematics classes. However, all the participants acknowledged the "inconsistency" of the function behavior with their initial expectations. One of the interviewed students believed that the interviewer switched the + and the - keys on the graphing calculator, in order to play a joke on him. For others, like Mitch, remembering "to do the opposite" was a common strategy for coping with perceived inconsistency.

Committing mathematics to memory in distinguishing the case of horizontal versus vertical translation was another issue mentioned by Mitch, which reoccurred in eight out of the ten interviews. Following the interviewer's prompting and an attempt to compare vertical and horizontal translations, participants focused on surface clues, such as "outside the bracket" or "in the bracket." For example, Ian explained: "The graph of $y$ equals $x$ minus three squared $\left[y=(x-3)^{2}\right]$ moves horizontally because three is in the bracket with $x$ instead of outside the bracket. And it moves right three because it's minus three in the bracket, and when in the bracket, it always moves opposite to the sign of the number."

Mitch is similar to other participating students in accepting the perceived inconsistency without a sincere attempt to understand its source. However, his explanation of following the pattern of $y=$ $(x-1)^{2}$ and $y=(x-2)^{2}$ in order to conclude the location of $y=(x-3)^{2}$ was echoed by only one other student in this group. A more common response was "I can't explain this, I just know how to do this."

In summary, there was no desire on the part of student-interviewees to understand or explain the phenomenon. When an explanation was requested, students presented rules to be memorized or generalizations from attending to patterns.

\subsection{Teachers' responses}

All the teachers participating in this study have sketched the graph of $y=(x-3)^{2}$ correctly. However, for the practicing teachers it was an immediate and effortless recall from memory, the way one would recall, rather than derive, a basic multiplication fact. Preservice teachers needed a few minutes of thinking and checking. It was evident that for some preservice teachers the horizontal translation of a parabola was not in their immediate repertoire of knowledge, but the location of the graph was derived correctly and without major effort.

Unlike the uniformity in students' tendency to rely on memorized rules, there was more variety in teachers' responses to the interviewer's request to explain the movement of the parabola. The explanations of preservice teachers did not differ in content or in assortment from those provided by practicing teachers. However, we note that when prompted by the interviewer, the majority of practicing teachers provided more than one explanation, while only four of the preservice teachers did the same. We sum- 
marize below several themes that emerged in the teachers' responses. Table 1 provides the frequency of occurrences of these themes.

\subsection{Citing rules}

A majority of the interviewed teachers (18 out of 31) referred to the "rule of horizontal translation." According to this "rule," $y=(x-3)^{2}$ has the same shape as $y=x^{2}$ but is located three units to the right. Thus, teachers' reliance on rules is indicative of students' reliance on memorizing these rules.

To reinforce memorization and to explain function translation to their students several practicing teachers have formulated "the law of opposites." They indicated that having "the law" helped their students in "getting it right." This, of course, raises issues with regard to the purpose and value of mathematics education. If the purpose is to "get it right" on an exam, then introducing such a law has its merit. However, if the purpose is to teach mathematical thinking, then the creation of such a law jeopardizes the consistency of mathematical structure and directs students' attention to memorization rather than to explanation.

\subsection{Point-wise approach}

Plugging numbers into the equation, creating a table of values, and then plotting the points seemed more convincing for some teachers than simply accepting what the computer or graphing calculator was showing. These teachers explained that they saw advantage in using the point-by-point creation of $y=(x-3)^{2}$ as an explanatory tool for their students. Interestingly though, a point-wise approach was not mentioned by any of the students. It appears that the utility and availability of graphing calculators and the lack of extensive experience with creating graphs manually, point by point, influences students' perception of graphs and suggests that the convincing power of teachers' explanations needs to be reexamined. Moreover, the influence of graphing calculators and graphing software on participants' explanations deserves the attention of further research.

\subsection{Attending to zero and "making up"}

Another common explanation, suggested by about a half of the participating teachers was to find the zero $(x=3)$, or the vertex, of the parabola and imply that the rest of the points are "symmetrically determined" around it. Those teachers were prompted to explain in what way the location of zero would determine the location of the rest of the points. In most cases, preservation of shape and symmetry were put forward as justifications. However, several teachers presented variations on the following explanation:

Table 1

Frequency of occurrences of themes in teachers' explanations

\begin{tabular}{llc}
\hline Theme in explanation & Preservice teachers $(n=15)$ & Practicing teachers $(n=16)$ \\
\hline Citing rules & 6 & 12 \\
Point-wise view/table of values & 4 & 7 \\
Attention to zero, "making up" & 7 & 8 \\
Transforming axes & 2 & 1
\end{tabular}


"In order to make up for the taking three away from $x$, we need to add three to each $x$-coordinate in order to get the same $y$-coordinates, so the graph shifts three to the right,"

or

"to get to a particular $y$-value, the $x$-value must be three greater, that is, farther to the right than originally, because three is being subtracted."

This was usually an extension of attending to the zero of the function and an attempt to explain "what happens to other points on a graph is the same as what happens to zero," without an explicit calculation. It was noted that explanations similar to these can help in justifying what happens after the result of translation is presented, rather than help in predicting the result.

Another explanation provided by a few teachers considered transformation of axes rather than transformation of a graph. These participants convinced themselves that $(x-3)$ actually meant moving the $y$-axis to the left, and therefore the parabola "looked as if" it moved to the right.

\subsection{A search for consistency}

It has been acknowledged that the direction of translation of parabola is inconsistent with intuitive expectations. This creates a dissonance for both students and teachers. This dissonance is further aggravated by the fact that the vertical translation operates "as expected," that is, the graph of the function $y=x^{2}+3$ is a vertical upward translation by three units of the graph of $y=x^{2}$. Therefore, the learners face not only a counterintuitive behavior of functions, but also inconsistency in the fact that some translations act according to their expectations while others do not.

The standard form in which the parabola is discussed in the local curriculum guide and the textbook is $(y-k)=a(x-h)^{2}$. It is stated that the value of $k$ determines the size of the vertical translation, while the value of $\mathrm{h}$ determines the size of the horizontal translation. However, the direction of the translation is omitted.

A repeated tendency of practicing teachers, most likely influenced by this general form, was to have their students consider the parabola $y=x^{2}-3$ as $y+3=x^{2}$. In this representation "adding 3 to $y$ " results in vertical translation the negative (downwards) direction. This view helps in achieving consistency with the horizontal translation, but does not provide an adequate explanation for the move of the graph in the "opposite" (that is, inconsistent with the initial expectation) direction.

In summary, teachers provided a variety of explanations, most common of which were citing the rules, considering the function point-wise and attending to the zero of the function. Most teachers were not completely satisfied with their explanations, but claimed to "have never seen a better one." As previously mentioned, there was no significant difference between practicing and preservice teachers in their explanations of the translation. However, preservice teachers' responses differed from the responses of practicing teachers on two accounts. The first is the ease of recall, acknowledged early in this section. Automatic and fluent retrieval is considered to be one of the indicators of expert knowledge (Bransford, Brown \& Cocking, 2000), and therefore it is not surprising that practicing teachers exhibited better expertise in the subject matter they taught than preservice teachers. Second, as expected by assuming expertise not only in content but also in a pedagogical content knowledge, practicing teachers, had a better understanding of the perceived inconsistency and of the problems that a horizontal translation presents to a learner. As a result, they had developed a larger repertoire of explanations as to why the horizontal translation of a function "behaves" as it does. 


\section{Analysis: understanding the routes}

"The central problem of education is not so much the description and categorization of the processes of development of knowledge, as the intervention into these processes" (Sierpinska, 1994, p. 121). However, description and categorization should precede the intervention, the way the diagnosis precedes the treatment. In what follows we describe what appears to be the cause of the problem of perceived inconsistency in translating a parabola, and suggest a pedagogical approach to transformations of functions.

The results indicate that the ability to determine the direction of horizontal translation correctly does not imply understanding of this function transformation and the ability to justify it. From the perspective of a learner, behavior of functions is counterintuitive. From the perspective of an observer, learners' intuition is misleading and it presents an obstacle to their understanding. In order to understand the sources of this intuition we turn our attention first to the notion of obstacles - cognitive, conceptual, and epistemological.

\subsection{Obstacles}

The notion of an epistemological obstacle was introduced by the French philosopher and scientist Gaston Bachelard. Bachelard described the development of scientific knowledge as constrained by intrinsic, and at times unrecognized, factors associated with the process of understanding, rather than by the complexities of the phenomena (Herscovics, 1989; Sierpinska, 1994). Herscovics (1989) reserved the term "epistemological obstacles" for obstacles that are encountered in the development of knowledge in a discipline. The obstacles in the conceptual development of an individual learner were denoted as "cognitive obstacles." Similar to Herscovics' cognitive obstacles, Zaslavsky (1997) introduced the notion of "conceptual obstacles," referring to "obstacles that have a cognitive nature that can be explained in terms of mathematical structures and concepts that underlie students' earlier learning experiences" (p. 20).

Sierpinska (1994) considered a broader interpretation of epistemological obstacles and described them as obstacles to some change in the frame of mind. This broader use of the term can be attributed to the historical development of knowledge in a discipline, as well as to the knowledge development of an individual learner. In this framework to learn means to overcome a difficulty. The construction of meaning is determined not only by moving towards the change, but also by various norms, beliefs and ways of thinking that constitute obstacles to this change.

Among the constraints identified by Bachelard as potential epistemological obstacles to the advancement of scientific thinking are influences of natural language, tendency to generalize and possibly deceptive intuitions (Norman \& Prichard, 1994). Though the notion of an epistemological obstacle was developed in the realm of science, it is applicable to the development of mathematical concepts, both in discipline and in individual learning. For example, the difficulty in accepting irrational quantities as numbers is an indication of an epistemological obstacle in the development of mathematics as a discipline. "Multiplication makes bigger" is a common example of a cognitive/conceptual obstacle in individual's knowledge acquisition. As beliefs and ways of thinking are influenced by prior experiences, this particular obstacle is based on experience with whole numbers, causing a difficulty when this experience is over-generalized to operations with fractions or integers.

In a similar fashion, turning to translation of functions, learners' experiences with numbers suggest that adding 3 to a variable results in a translation in the positive direction (right), while subtracting 3 (or adding negative 3 ) results in translation in the negative direction (left). On the surface, such a view is 
an over-generalization of prior experience of adding and subtracting numbers on the number line. In the next section we suggest a more profound and detailed explanation of learner's cognition.

\subsection{Reducing abstraction}

We believe that a possible source of a learner's obstacle is in trying to see (b) $y=(x-3)^{2}$ as a transformation of (a) $y=x^{2}$, and in doing so creating confusion between the function defining the parabola and the function of transformation. It is evident from the data that students' focus is on the algebraic representation of functions. Comparing the algebraic representation $y=x^{2}$ and $y=(x-3)^{2}$, students correctly notice that (b) is derived from (a) by substituting $(x-3)$ in place of $x$. As mentioned earlier, this interpretation is extended, mostly intuitively, to the view of the number line: for any number $x, x-3$ is located three units to the left of $x$. We suggest that the main source of difficulty here is in seeing this algebraic replacement as a transformation ( $x$ moves to $x-3)$ and trying to infer the geometric transformation, the movement of the graph, from the algebraic substitution. That is to say that the transformation $f(x) \mapsto f(x-3)$ is simplified to be viewed as $x \Leftrightarrow(x-3)$. Such a view is in accord with what Hazzan (1999) described as reducing abstraction, which is a strategy used by learners to cope with complexity. One of the strands of reducing abstraction described by Hazzan is in students' attention to a simpler object in place of a more complex one. In her example, some students' difficulties with Abstract Algebra can be explained by focusing on one of the elements in a set rather than on a set itself. In our example, students' attention is on an object of a variable $(x)$ rather than on a more abstract object of a function $f(x)$.

\subsection{Pedagogical obstacle?}

Though the studies of Eisenberg and Dreyfus (1994) and Baker et al. (2000) agree on the difficulty presented by horizontal transformation, they differ in the way they explain the source of this difficulty. Eisenberg and Dreyfus see the difficulty in the visual processing of information. Baker et al. attribute the difficulty to the complexity of mental construction needed to process a horizontal transformation. We described above how students' difficulty can be attributed to a conceptual-cognitive-epistemological obstacle and to human tendency to reduce the abstraction level when facing complexity.

However, there is another possible source of the difficulty, and it is in the instructional sequence in which the discussion of transformation of functions takes place. The focus of students and teachers on algebraic representation is not their fault and may not be their choice. This choice is prescribed by a traditional curricular approach to function transformations. This approach embeds the discussion of transformations of parabolas, quadratic relations, or polynomial functions in the general context of functions, rather than in the context of transformations. This standard pedagogical approach could be reinforcing the obstacle, rather than trying to overcome it. Our belief is that the problem can be avoided, or at least minimized, by a pedagogical approach that situates transformations of functions in the context of transformations. We outline this approach below.

\section{Pedagogical approach: rerouting}

As stated above, we believe that one of the difficulties that learners have in understanding horizontal transformations of a function is a consequence of the instructional sequence in which the treatment of 
transformations of functions is presented in the context of exploring functions, rather than in the context of exploring transformations. In order to situate the discussion of translations of functions in the context of transformations, we must first create this context for the learners.

\subsection{Creating a context}

To "apply transformations and use symmetry to analyze mathematical situations" is one of the Geometry standards identified by the NCTM for K-12 (NCTM, 2002). The progressive sequence of expectations for students outlined by the NCTM includes the following:

- recognize and apply slides, flips, and turns (Grades PreK-2; p. 96);

- predict and describe results of sliding, flipping and turning two dimensional shapes (Grades 3-5; p. 164);

- describe sizes positions and orientations of shapes under informal transformations such as flips, turns, slides and scaling (Grades 6-8; p. 232);

- understand and represent translations, reflections, rotations and dilations of objects in the plane by using sketches, coordinates, vectors, function notation, and matrices (Grades 9-12; p. 308).

That is, students' early encounters with transformations usually take place in elementary school, when rigid motions of objects - slides, turns, and flips — are introduced. Those motions are explored in further detail throughout the grades and later formalized as translations (defined by a vector), rotations (defined by a center point and an angle) and reflections (defined by a line). It is the function notation briefly mentioned by the NCTM in the geometry standard for Grades 9-12 that we wish to focus on here. Although we focus on translations, as this is the concern of this article, a similar approach can be extended to other transformations as well.

\subsection{Translation on a coordinate plane}

Translation on a plane is defined by a vector (or directed segment) that specifies the direction of the motion and the distance. It is our experience that students tend to identify translations by breaking this motion into its horizontal and vertical components. This view leads to the natural introduction of the formal notation for translation

$$
(x, y) \mapsto(x+a, y+b) \quad \text { or } \quad T((x, y))=(x+a, y+b),
$$

where $a$ and $b$ are horizontal and vertical components of the motion, respectively. This function notation for a transformation is often referred to as a mapping rule. Fig. 1 illustrates the effect of $T((x, y))=$ $(x+5, y-3)$ on a set of points in a triangle; the pre-image appears in black and the image in gray.

Once function notation is introduced, students should be given ample opportunity to connect the visual image of translation to the mapping rule. This is achieved by carrying out transformations according to given mappings as well as by identifying mappings according to given visual images. Specifically, students will identify positive and negative values of " $a$ " with motion to the right or to the left, respectively; positive and negative values of " $b$ " with motion up and down, respectively. Furthermore, they will associate the strict horizontal motion with $b=0$ and strict vertical motion with $a=0$.

Since any set of points can be translated according to the mapping rule, this set of points can be a parabola. This is our focus in the next section. 


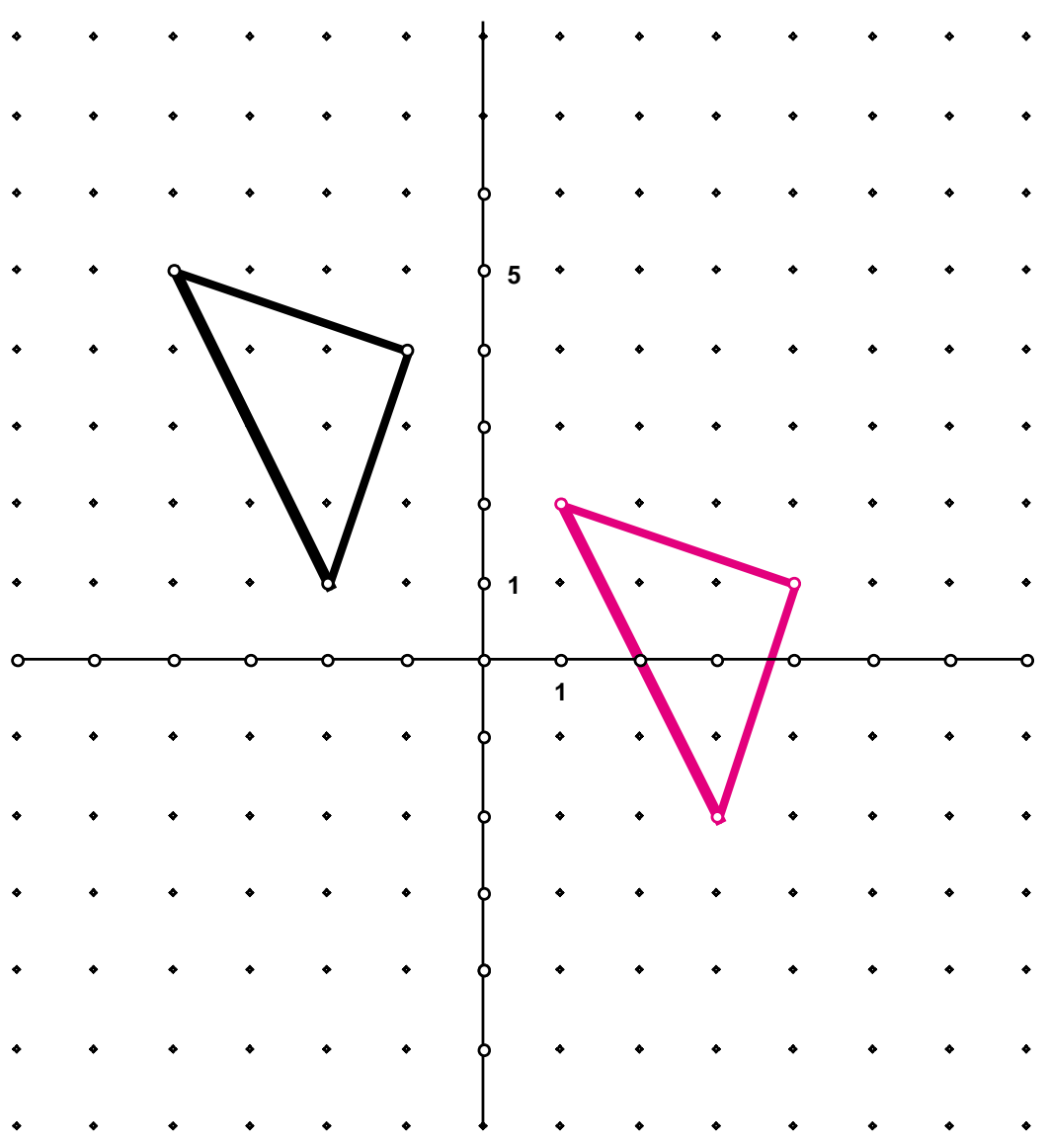

Fig. 1. Applying $T((x, y))=(x+5, y-3)$ on the set of points of a triangle.

\subsection{Translating a parabola}

As mentioned above, we wish to situate the discussion of transformations of functions in the context of transformations. We start by exploring a translation of a parabola $y=x^{2}$. Given the experiences described in the previous section, students understand that $T((x, y))=(x+3, y)$ represents a horizontal translation by three units to the right. Now restrict the set of points $(x, y)$ to a canonical parabola and apply $T$ to it. Fig. 2 illustrates the effect of $T((x, y))=(x+3, y)$ on a set of points of a canonical parabola; the pre-image appears in black and the image in gray.

The task now becomes connecting the translation image to its algebraic representation. Recall that the set of points of the source is described by $y=x^{2}$. Without loss of generality, focus on a point $(a, b)$ of the source set that was translated to the point $(c, d)$ of the image set. According to the specific translation performed, $d=b$ and $c=a+3$. We wish to connect $c$ and $d$ in an equation. Relating $c$ to $d$, we obtain the following: $d=b$ and $c=a+3$ which implies $a=c-3$. However, $b=a^{2}$ as $(a, b)$ is a point on the source parabola. Substitution leads to $d=(c-3)^{2}$. Since the above is true for every point of the image set, the image of the translation is described by the equation $y=(x-3)^{2}$. Of course, one can work 


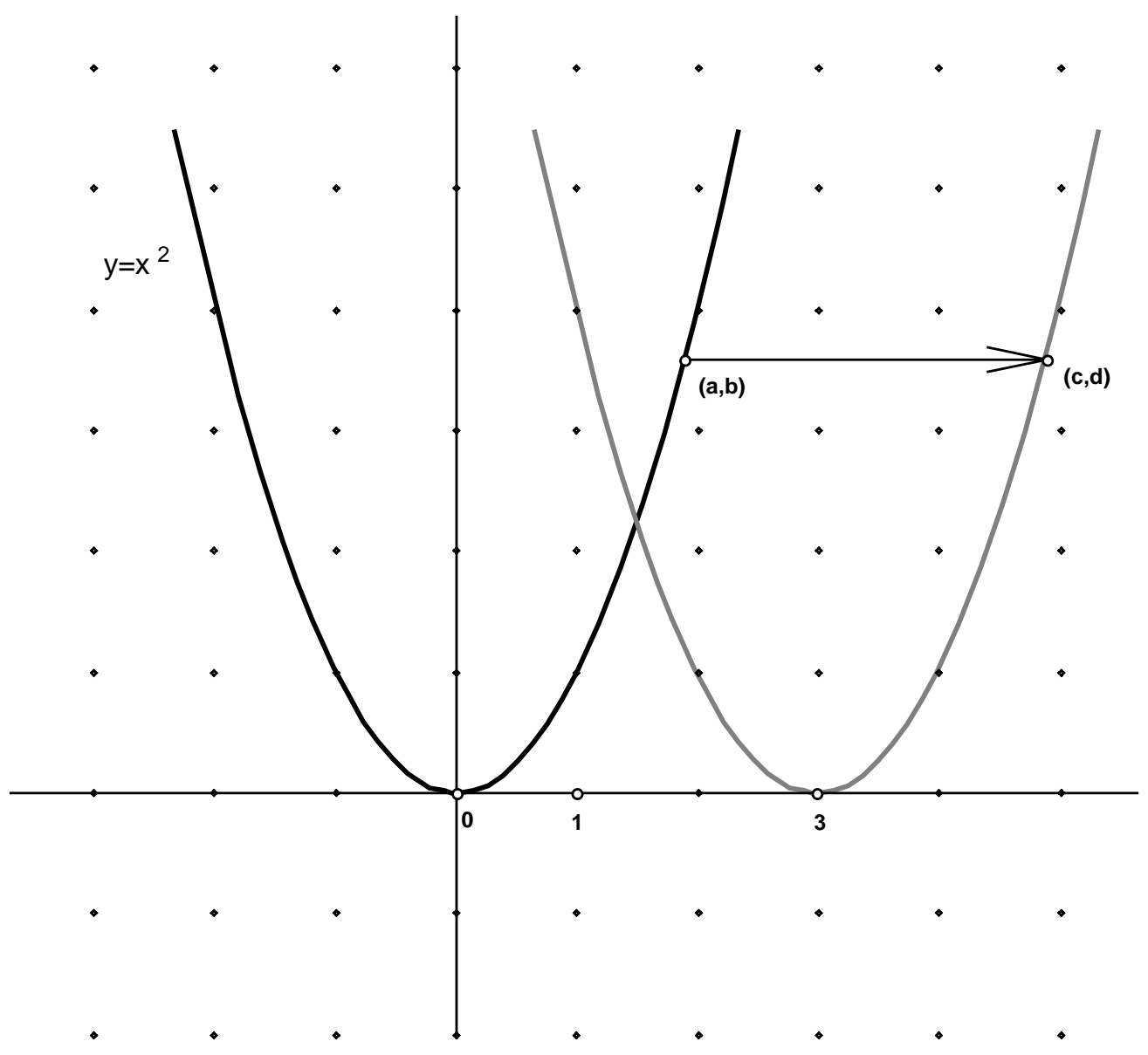

Fig. 2. Applying $T((x, y))=(x+3, y)$ on the graph of $y=x^{2}$.

directly with $x$ 's and $y$ 's, but by switching to $a$ 's, $b$ 's, and $c^{\prime}$ 's, we believe it can be easier for students. This explains the "unexpected" appearance of " -3 " in the horizontal translation to the right.

This method can be also used for transformations that are not "counterintuitive" or "problematic." Consider the same approach applied to a vertical translation $T((x, y))=(x, y+3)$. Focus on a point $(a$, $b$ ) of the source that is translated to point $(c, d)$ of the image (Fig. 3). In this case, $a=c$ and $d=b+3$. Points of the source satisfy $b=a^{2}$. Therefore, $d=b+3=a^{2}+3=c^{2}+3$. Since this is true for any point on the image, the set of image points is described by $y=x^{2}+3$ as expected. Fig. 3 illustrates the effect of $T((x, y))=(x, y+3)$ on a set of points of a canonical parabola; the pre-image appears in black and the image in gray.

Consideration of a geometric transformation as a starting point is not limited to translations. We envision an approach in which students explore a variety of transformations, making a connection between the conventional definition of a transformation and its effect on specific sets of points on the plane. Such sets could be simple geometric shapes in the initial stages of exploration and graphs of specific functions or relationships in later stages. 


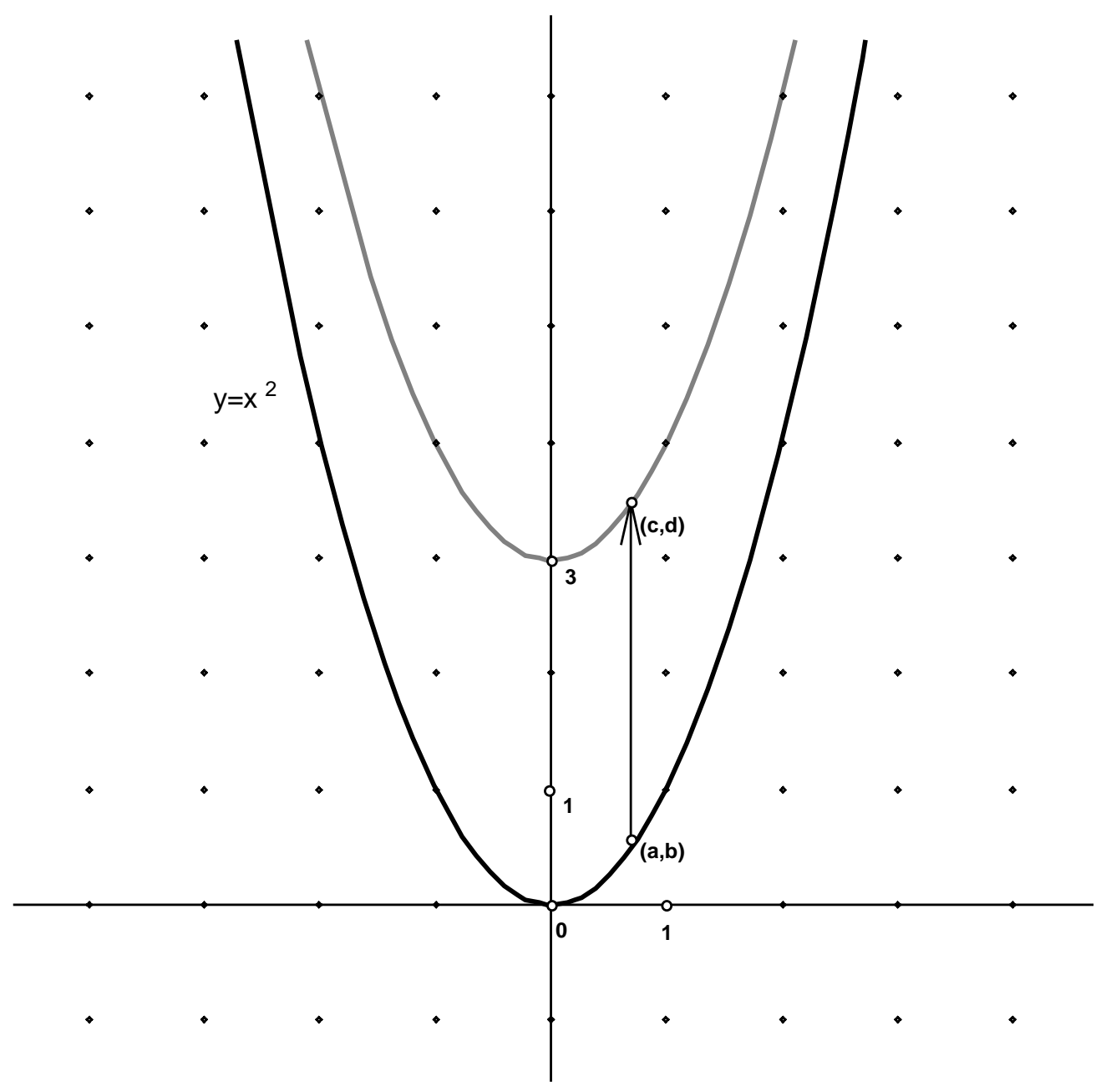

Fig. 3. Applying $T((x, y))=(x, y+3)$ on the graph of $y=x^{2}$.

\subsection{Rerouting}

A popular recommendation to help learners overcome their misconceptions or misleading intuition is to engage them in situations where their initial conceptions and intuitions fail. Research shows that learners do not give up their intuitive beliefs easily, and may possess different, and at times conflicting, conceptions (Fischbein, 1987).

The goal of our pedagogical approach is not to change students' minds about the direction of horizontal translation. Our goal is to balance the dissonance presented by the discord between initial intuition and graphical image. This is done successfully with the use of graphing devices, either electronic or manual.

"Overcoming an obstacle" creates a metaphorical mental image of climbing a hill to get to the other side. However, rather than overcoming an obstacle, the right question might be, Can it be avoided? Extending the metaphor, can we get to the other side by going around the hill? We believe it is possible, 
and we consider the approach suggested here as "rerouting." Specifically, we propose a route to function translation via consideration of transformations, rather than a conventional consideration of function translations derived from the algebraic representation of functions.

\section{Epilogue}

Future research will determine whether the intervention or instructional sequence presented above is helpful for students' understanding of the translation of a parabola in particular and of any function in general. So far, we have explored such an intervention on a smaller scale, presenting it to teachers as an alternative pedagogical approach. The participating teachers referred to this view of transformations as "an eye opening clarification" or a "pedagogical AHA!"

\section{References}

Asiala, M., Brown, A., DeVries, D. J., Dubinsky, E., Mathews, D., \& Thomas, K. (1996). A framework for research and curriculum development in undergraduate mathematics education. In: E. Dubinsky, A. Schoenfeld, \& J. Kaput (Eds.), Research in collegiate mathematics education (Vol. 2, pp. 1-32). Providence, RI: American Mathematical Society.

Baker, B., Hemenway, C., \& Trigueros, M. (2000). On transformations of basic functions. In: H. Chick, K. Stacey, \& J. Vincent (Eds.), Proceedings of the 12th ICMI Study Conference on the Future of the Teaching and Learning of Algebra (Vol. 1, pp. 41-47). University of Melbourne.

Borba, M. C., \& Confrey, J. (1996). A students' construction of transformations of functions in a multirepresentational environment. Educational Studies in Mathematics, 31(3), 319-337.

Bransford, J. D., Brown, A. L., \& Cocking, R. R. (2000). How people learn: brain, mind, experience and school. Washington, DC: National Academy Press.

Dubinsky, E., \& MacDonald, M. (2001). APOS: a constructivist theory of learning in undergraduate mathematics education research. In: D. Holton (Ed.), The Teaching and Learning of Mathematics at University Level: An ICMI Study (pp. 273-280). Dordrecht: Kluwer Academic Publishers.

Eisenberg, T., \& Dreyfus, T. (1994). On understanding how students learn to visualize function transformations. In: E. Dubinsky, A. Schoenfeld, \& J. Kaput (Eds.), Research in collegiate mathematics education (Vol. 1, pp. 45-68). Providence, RI: American Mathematical Society.

Fischbein, E. (1987). Intuition in science and mathematics: an educational approach. Dordrecht, Netherlands: Reidel.

Hazzan, O. (1999). Reducing abstraction level when learning abstract algebra concepts. Educational Studies in Mathematics, 40(1), 71-90.

Herscovics, N. (1989). Cognitive obstacles encountered in the learning of Algebra. In: C. Kieran, \& S. Wagner (Eds.), Research issues in the learning and teaching of algebra (pp. 60-86). Hillsdale, NJ: Lawrence Erlbaum Associates.

National Council of Teachers of Mathematics (NCTM). (2002). Principles and standards for school mathematics. Reston, VA: NCTM.

Norman, F. A., \& Prichard, M. K. (1994). Cognitive obstacles to the learning of calculus: a Krutetskian perspective. In: J. Kaput, \& E. Dubinsky (Eds.), Research issues in undergraduate mathematics learning: preliminary analyses and results. MAA notes number 33. Mathematical Association of America.

Sierpinska, A. (1994). Understanding in mathematics. London: Falmer Press.

Zaslavsky, O. (1997). Conceptual obstacles in the learning of quadratic functions. Focus on Learning Problems in Mathematics, 19(1), 20-44. 